\title{
Angioimmunoblastic T-Cell Lymphoma
}

National Cancer Institute

\section{Source}

National Cancer Institute. Angioimmunoblastic T-Cell Lymphoma. NCI Thesaurus. Code C7528.

A peripheral T-cell lymphoma of mature $T$ follicular helper ( $T F H$ ) cells characterized by systemic disease and a polymorphous infiltrate involving lymph nodes, with a prominent proliferation of high endothelial venules and follicular dendritic cells. EBV-positive cells are nearly always present. It is a clinically aggressive lymphoma and seen mainly in older adults. (WHO 2017) 